\title{
General Theory of Quasi-Commutative BCI-algebras
}

\author{
Tao Sun \\ Qingdao University of Science \\ and Technology \\ China \\ Chenglong $\mathrm{Wu}$ \\ Qingdao University of Science \\ and Technology \\ China
}

\author{
Weibo Pan \\ Qingdao University of Science \\ and Technology \\ China \\ Xiquan Liang \\ Qingdao University of Science \\ and Technology \\ China
}

Summary. It is known that commutative BCK-algebras form a variety, but BCK-algebras do not [4]. Therefore H. Yutani introduced the notion of quasicommutative BCK-algebras. In this article we first present the notion and general theory of quasi-commutative BCI-algebras. Then we discuss the reduction of the type of quasi-commutative BCK-algebras and some special classes of quasicommutative BCI-algebras.

MML identifier: $\underline{\text { BCIALG_5 }}$, version: $\underline{7.9 .014 .103 .1019}$

The articles [7], [2], [3], [1], [5], and [6] provide the terminology and notation for this paper.

Let $X$ be a BCI-algebra, let $x, y$ be elements of $X$, and let $m, n$ be elements of $\mathbb{N}$. The functor $\operatorname{Polynom}(m, n, x, y)$ yields an element of $X$ and is defined as follows:

(Def. 1) $\operatorname{Polynom}(m, n, x, y)=\left((x \backslash(x \backslash y))^{m+1} \backslash(y \backslash x)\right)^{n}$.

We adopt the following convention: $X$ denotes a BCI-algebra, $x, y, z$ denote elements of $X$, and $i, j, k, l, m, n$ denote elements of $\mathbb{N}$.

One can prove the following propositions:

(1) If $x \leq y \leq z$, then $x \leq z$.

(2) If $x \leq y \leq x$, then $x=y$. 
(3) For every BCK-algebra $X$ and for all elements $x, y$ of $X$ holds $x \backslash y \leq x$ and $(x \backslash y)^{n+1} \leq(x \backslash y)^{n}$.

(4) For every BCK-algebra $X$ and for every element $x$ of $X$ holds $\left(0_{X} \backslash x\right)^{n}=$ $0_{X}$.

(5) For every BCK-algebra $X$ and for all elements $x, y$ of $X$ such that $m \geq n$ holds $(x \backslash y)^{m} \leq(x \backslash y)^{n}$.

(6) Let $X$ be a BCK-algebra and $x, y$ be elements of $X$. Suppose $m>n$ and $(x \backslash y)^{n}=(x \backslash y)^{m}$. Let $k$ be an element of $\mathbb{N}$. If $k \geq n$, then $(x \backslash y)^{n}=$ $(x \backslash y)^{k}$.

(7) $\operatorname{Polynom}(0,0, x, y)=x \backslash(x \backslash y)$.

(8) $\operatorname{Polynom}(m, n, x, y)=\left((\operatorname{Polynom}(0,0, x, y) \backslash(x \backslash y))^{m} \backslash(y \backslash x)\right)^{n}$.

(9) $\operatorname{Polynom}(m+1, n, x, y)=\operatorname{Polynom}(m, n, x, y) \backslash(x \backslash y)$.

(10) $\operatorname{Polynom}(m, n+1, x, y)=\operatorname{Polynom}(m, n, x, y) \backslash(y \backslash x)$.

(11) $\operatorname{Polynom}(n+1, n+1, y, x) \leq \operatorname{Polynom}(n, n+1, x, y)$.

(12) $\operatorname{Polynom}(n, n+1, x, y) \leq \operatorname{Polynom}(n, n, y, x)$.

Let $X$ be a BCI-algebra. We say that $X$ is quasi-commutative if and only if:

(Def. 2) There exist elements $i, j, m, n$ of $\mathbb{N}$ such that for all elements $x, y$ of $X$ holds $\operatorname{Polynom}(i, j, x, y)=\operatorname{Polynom}(m, n, y, x)$.

Let us observe that BCI-EXAMPLE is quasi-commutative.

One can check that there exists a BCI-algebra which is quasi-commutative.

Let $i, j, m, n$ be elements of $\mathbb{N}$. A BCI-algebra is called a BCI-algebra commutating with $i, j$ and $m, n$ if:

(Def. 3) For all elements $x, y$ of it holds $\operatorname{Polynom}(i, j, x, y)=\operatorname{Polynom}(m, n, y, x)$.

One can prove the following propositions:

(13) $X$ is a BCI-algebra commutating with $i, j$ and $m, n$ if and only if $X$ is a BCI-algebra commutating with $m, n$ and $i, j$.

(14) Let $X$ be a BCI-algebra commutating with $i, j$ and $m, n$ and $k$ be an element of $\mathbb{N}$. Then $X$ is a BCI-algebra commutating with $i+k, j$ and $m$, $n+k$.

(15) Let $X$ be a BCI-algebra commutating with $i, j$ and $m, n$ and $k$ be an element of $\mathbb{N}$. Then $X$ is a BCI-algebra commutating with $i, j+k$ and $m+k, n$.

One can verify that there exists a BCK-algebra which is quasi-commutative.

Let $i, j, m, n$ be elements of $\mathbb{N}$. One can check that there exists a BCI-algebra commutating with $i, j$ and $m, n$ which is BCK-5.

Let $i, j, m, n$ be elements of $\mathbb{N}$. A BCK-algebra commutating with $i, j$ and $m, n$ is BCK-5 BCI-algebra commutating with $i, j$ and $m, n$.

One can prove the following propositions: 
(16) $X$ is a BCK-algebra commutating with $i, j$ and $m, n$ if and only if $X$ is a BCK-algebra commutating with $m, n$ and $i, j$.

(17) Let $X$ be a BCK-algebra commutating with $i, j$ and $m, n$ and $k$ be an element of $\mathbb{N}$. Then $X$ is a BCK-algebra commutating with $i+k, j$ and $m, n+k$.

(18) Let $X$ be a BCK-algebra commutating with $i, j$ and $m, n$ and $k$ be an element of $\mathbb{N}$. Then $X$ is a BCK-algebra commutating with $i, j+k$ and $m+k, n$.

(19) For every BCK-algebra $X$ commutating with $i, j$ and $m, n$ and for all elements $x, y$ of $X$ holds $(x \backslash y)^{i+1}=(x \backslash y)^{n+1}$.

(20) For every BCK-algebra $X$ commutating with $i, j$ and $m, n$ and for all elements $x, y$ of $X$ holds $(x \backslash y)^{j+1}=(x \backslash y)^{m+1}$.

(21) Every BCK-algebra commutating with $i, j$ and $m, n$ is a BCK-algebra commutating with $i, j$ and $j, n$.

(22) Every BCK-algebra commutating with $i, j$ and $m, n$ is a BCK-algebra commutating with $n, j$ and $m, n$.

Let us consider $i, j, m, n$. The functor $\min (i, j, m, n)$ yielding an extended real number is defined as follows:

(Def. 4) $\min (i, j, m, n)=\min (\min (i, j), \min (m, n))$.

The functor $\max (i, j, m, n)$ yielding an extended real number is defined by:

(Def. 5) $\max (i, j, m, n)=\max (\max (i, j), \max (m, n))$.

Next we state a number of propositions:

(23) $\min (i, j, m, n)=i$ or $\min (i, j, m, n)=j$ or $\min (i, j, m, n)=m$ or $\min (i, j, m, n)=n$.

(24) $\max (i, j, m, n)=i$ or $\max (i, j, m, n)=j$ or $\max (i, j, m, n)=m$ or $\max (i, j, m, n)=n$.

(25) If $i=\min (i, j, m, n)$, then $i \leq j$ and $i \leq m$ and $i \leq n$.

(26) $\max (i, j, m, n) \geq i$ and $\max (i, j, m, n) \geq j$ and $\max (i, j, m, n) \geq m$ and $\max (i, j, m, n) \geq n$.

(27) Let $X$ be a BCK-algebra commutating with $i, j$ and $m, n$. Suppose $i=\min (i, j, m, n)$. If $i=j$, then $X$ is a BCK-algebra commutating with $i, i$ and $i, i$.

(28) Let $X$ be a BCK-algebra commutating with $i, j$ and $m, n$. Suppose $i=\min (i, j, m, n)$. Suppose $i<j$ and $i<n$. Then $X$ is a BCK-algebra commutating with $i, i+1$ and $i, i+1$.

(29) Let $X$ be a BCK-algebra commutating with $i, j$ and $m$, $n$. Suppose $i=\min (i, j, m, n)$. Suppose $i<j$ and $i=n$ and $i=m$. Then $X$ is a BCK-algebra commutating with $i, i$ and $i, i$. 
(30) Let $X$ be a BCK-algebra commutating with $i, j$ and $m$, $n$. Suppose $i=\min (i, j, m, n)$. Suppose $i<j$ and $i=n$ and $i<m<j$. Then $X$ is a BCK-algebra commutating with $i, m+1$ and $m, i$.

(31) Let $X$ be a BCK-algebra commutating with $i, j$ and $m$, $n$. Suppose $i=\min (i, j, m, n)$. Suppose $i<j$ and $i=n$ and $j \leq m$. Then $X$ is a BCK-algebra commutating with $i, j$ and $j, i$.

(32) Let $X$ be a BCK-algebra commutating with $i, j$ and $m, n$. Suppose $l \geq j$ and $k \geq n$. Then $X$ is a BCK-algebra commutating with $k, l$ and $l, k$.

(33) Let $X$ be a BCK-algebra commutating with $i, j$ and $m, n$. Suppose $k \geq \max (i, j, m, n)$. Then $X$ is a BCK-algebra commutating with $k, k$ and $k, k$.

(34) Let $X$ be a BCK-algebra commutating with $i, j$ and $m, n$. Suppose $i \leq m$ and $j \leq n$. Then $X$ is a BCK-algebra commutating with $i, j$ and $i$, $j$.

(35) Let $X$ be a BCK-algebra commutating with $i, j$ and $m$, $n$. Suppose $i \leq m$ and $i<n$. Then $X$ is a BCK-algebra commutating with $i, j$ and $i$, $i+1$.

(36) If $X$ is a BCI-algebra commutating with $i, j$ and $j+k, i+k$, then $X$ is a BCK-algebra.

(37) $X$ is a BCI-algebra commutating with 0,0 and 0,0 if and only if $X$ is a BCK-algebra commutating with 0,0 and 0,0 .

(38) $X$ is a commutative BCK-algebra iff $X$ is a BCI-algebra commutating with 0,0 and 0,0 .

Let $X$ be a BCI-algebra. We introduce $p$-Semisimple-part $X$ as a synonym of AtomSet $X$.

In the sequel $B, P$ are non empty subsets of $X$.

One can prove the following propositions:

(39) For every BCI-algebra $X$ such that $B=$ BCK-part $X$ and $P=$ $p$-Semisimple-part $X$ holds $B \cap P=\left\{0_{X}\right\}$.

(40) For every BCI-algebra $X$ such that $P=p$-Semisimple-part $X$ holds $X$ is a BCK-algebra iff $P=\left\{0_{X}\right\}$.

(41) For every BCI-algebra $X$ such that $B=$ BCK-part $X$ holds $X$ is a $p$ semisimple BCI-algebra iff $B=\left\{0_{X}\right\}$.

(42) If $X$ is a $p$-semisimple BCI-algebra, then $X$ is a BCI-algebra commutating with 0,1 and 0,0 .

(43) Suppose $X$ is a $p$-semisimple BCI-algebra. Then $X$ is a BCI-algebra commutating with $n+j, n$ and $m, m+j+1$.

(44) Suppose $X$ is an associative BCI-algebra. Then $X$ is a BCI-algebra commutating with 0,1 and 0,0 and a BCI-algebra commutating with 1, 0 and 0,0 . 
(45) Suppose $X$ is a weakly-positive-implicative BCI-algebra. Then $X$ is a BCI-algebra commutating with 0,1 and 1,1 .

(46) If $X$ is a positive-implicative BCI-algebra, then $X$ is a BCI-algebra commutating with 0,1 and 1,1 .

(47) If $X$ is an implicative BCI-algebra, then $X$ is a BCI-algebra commutating with 0,1 and 0,0 .

(48) If $X$ is an alternative BCI-algebra, then $X$ is a BCI-algebra commutating with 0,1 and 0,0 .

(49) $X$ is a BCK-positive-implicative BCK-algebra if and only if $X$ is a BCKalgebra commutating with 0,1 and 0,1 .

(50) $X$ is a BCK-implicative BCK-algebra iff $X$ is a BCK-algebra commutating with 1,0 and 0,0 .

One can check that every BCK-algebra which is BCK-implicative is also commutative and every BCK-algebra which is BCK-implicative is also BCKpositive-implicative.

The following propositions are true:

(51) $X$ is a BCK-algebra commutating with 1,0 and 0,0 if and only if $X$ is a BCK-algebra commutating with 0,0 and 0,0 and a BCK-algebra commutating with 0,1 and 0,1 .

(52) Let $X$ be a quasi-commutative BCK-algebra. Then $X$ is a BCK-algebra commutating with 0,1 and 0,1 if and only if for all elements $x, y$ of $X$ holds $x \backslash y=x \backslash y \backslash y$.

(53) Let $X$ be a quasi-commutative BCK-algebra. Then $X$ is a BCK-algebra commutating with $n, n+1$ and $n, n+1$ if and only if for all elements $x$, $y$ of $X$ holds $(x \backslash y)^{n+1}=(x \backslash y)^{n+2}$.

(54) If $X$ is a BCI-algebra commutating with 0,1 and 0,0 , then $X$ is a BCI-commutative BCI-algebra.

(55) If $X$ is a BCI-algebra commutating with $n, 0$ and $m, m$, then $X$ is a BCI-commutative BCI-algebra.

(56) Let $X$ be a BCK-algebra commutating with $i, j$ and $m, n$. Suppose $j=0$ and $m>0$. Then $X$ is a BCK-algebra commutating with 0,0 and 0,0 .

(57) Let $X$ be a BCK-algebra commutating with $i, j$ and $m$, $n$. Suppose $m=0$ and $j>0$. Then $X$ is a BCK-algebra commutating with 0,1 and 0,1 .

(58) Let $X$ be a BCK-algebra commutating with $i, j$ and $m, n$. Suppose $n=0$ and $i \neq 0$. Then $X$ is a BCK-algebra commutating with 0,0 and 0,0 .

(59) Let $X$ be a BCK-algebra commutating with $i, j$ and $m, n$. Suppose $i=0$ and $n \neq 0$. Then $X$ is a BCK-algebra commutating with 0,1 and 0,1 . 


\section{REFERENCES}

[1] Grzegorz Bancerek. The ordinal numbers. Formalized Mathematics, 1(1):91-96, 1990.

[2] Yuzhong Ding. Several classes of BCI-algebras and their properties. Formalized Mathematics, 15(1):1-9, 2007.

[3] Yuzhong Ding and Zhiyong Pang. Congruences and quotient algebras of BCI-algebras. Formalized Mathematics, 15(4):175-180, 2007.

[4] Jie Meng and YoungLin Liu. An Introduction to BCI-algebras. Shaanxi Scientific and Technological Press, 2001.

[5] Tao Sun, Dahai Hu, and Xiquan Liang. Several classes of BCK-algebras and their properties. Formalized Mathematics, 15(4):237-242, 2007.

[6] Andrzej Trybulec and Agata Darmochwał. Boolean domains. Formalized Mathematics, 1(1):187-190, 1990.

[7] Zinaida Trybulec. Properties of subsets. Formalized Mathematics, 1(1):67-71, 1990.

Received May 13, 2008 